\title{
Jejunal obstruction due to currency notes
}

D. Seneviratne Epa , D.C. Palkumbura, G. Goonetilleke

Sri Jayewardenepura General Hospital and Postgraduate Teaching Institute, Nugegoda, Sri Lanka

Key words : Jejunal obstruction, foreign body, laparotomy

\section{Introduction}

We report a case of jejunal obstruction due to ingestion of currency notes in a patient with a psychiatric history. Both the site and cause of intestinal obstruction in this case is unusual.

\section{Case Report}

A 65 year old lady with a history of depression, presented with vomiting and generalized abdominal pain for three weeks, increasing in severity over three days, with no alteration of bowel habits, per rectal bleeding, loss of appetite or weight loss. There was no history of swallowing any foreign body. Abdomen was mildly distended, but was soft and non-tender. Bowel sounds were normal. Basic haematological and biochemical investigations were normal. Erect x-ray abdomen showed an elongated opacity adjacent to the tip of the transverse process of the second lumbar vertebra. Patient was managed symptomatically with initial improvement.

However on third day, she developed repeated bilious vomiting with colicky abdominal pain. Bowels remained open and abdominal signs didn't change either. Repeat erect $\mathrm{x}$-ray abdomen showed multiple air fluid levels with the shifting of the previously noted opacity to a point adjacent to lateral border of third lumbar vertebral body. A nasogastric tube was inserted and intravenous fluids given. However, symptoms recurred on fifth day, after removal of the nasogastric tube and resumption of feeding. At this stage, an exploratory laparotomy was performed and an intraluminal mass was noted about one foot from the duodeno-jejunal junction, with dilated proximal jejunal loops. Enterotomy was done and an approximately Correspondence: G.Goonetilleke E-mail: drgcsr@sltnet.lk
$5 \times 5 \times 5 \mathrm{~cm}$ cuboidal, irregular, firm mass, which was not attached to the walls of the jejunal lumen was extracted(Fig 1). It was covered with a thick layer of faecal matter and weighed $100 \mathrm{~g}$. Within the mass were several folded currency notes which were fragmented and defaced beyond recognition. However there was one ten rupee Sri Lankan currency note in a recognizable state(Fig 2). Rest of the laparotomy findings were unremarkable. She made an uneventful postoperative recovery and all symptoms resolved.

\section{Discussion}

Our patient developed recurrent bilious vomiting and colicky abdominal pain, but continued to open bowel and had only mild distension. This is the expected presentation of a high intestinal obstruction which predominately causes vomiting rather than distension or constipation(1). Jejunum however is an unusual site of intraluminal obstruction, but has been reported due to gall stones and haematomas(2).

Intestinal obstruction due to foreign body ingestion, usually by accident, has been reported especially among children and psychiatric patients(3). Trichobezoar due to repeated swallowing of hair, causing intestinal obstruction, is well documented among psychiatric patients(4). Our patient who also had a psychiatric history denied ingestion of a foreign body either accidental or intentional. This is probably the first report of such obstruction due to swallowing of currency notes. She was referred for psychiatric assessment with a view to avoid possible recurrence in this back ground.

The majority of ingested foreign bodies pass through the gastrointestinal tract without complications and only a minority of cases require surgical intervention for complications(5). Though our patient was initially managed conservatively, the recurrence of symptoms led to surgical intervention and a successful outcome. 


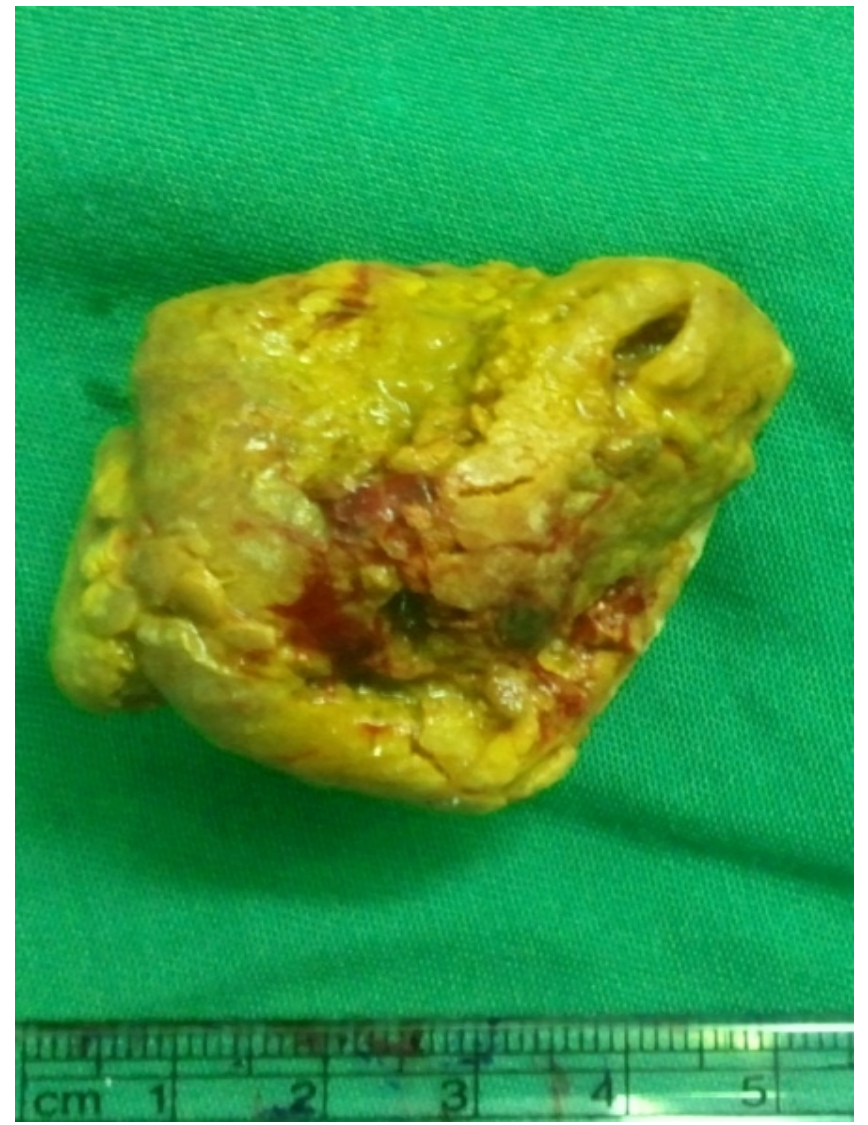

Figure 1. The obstructing mass

\section{References}

1. Baines MJ. ABC of palliative care. Nausea, vomiting, and intestinal obstruction. British Medical Journal 1997; 35: 1148-1150

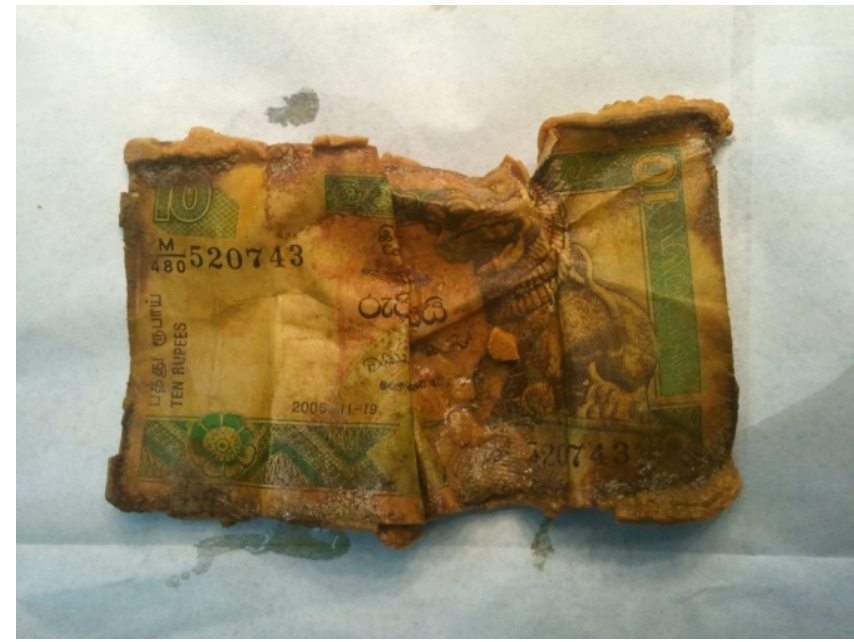

Figure 2. The currency note

2. Davis DR, Thomas CY. Intramural Hematoma of the Duodenum and Jejunum: A Cause of High Intestinal Obstruction-Report of Three Cases due to Trauma. Annals of Surgery 1961 Mar; 153(3):394-8.

3. Schwartz GF, Polsky HS: Ingested foreign bodies of the gastrointestinal tract. Am Surgeon 1976; 42:236-8.

4. Gaujoux S, Bach G, Au J, Godiris-Petit G, Munoz-

Bongrand N, Cattan P, SarfatiE: Trichobezoar: A rare cause of bowel obstruction. World J Gastrointest Surg 2011 April 27; 3(4): 54-5.

5. Gracia C, Frey CF, Bodai BI. Diagnosis and management of foreign bodies: a ten-year experience. Ann Emerg Med 1984 Jan; 13(1): 30-4

\section{Key points:}

- Intramural obstruction of jejunum is rare.

- Foreign body ingestion is a potential cause.

- If conservative measures fail surgery is mandatory.

- The cause is usually detected at surgery. 